\title{
Sociodemographic and clinical predictors of compliance with antidepressants for depressive disorders: systematic review of observational studies
}

\author{
This article was published in the following Dove Press journal: \\ Patient Preference and Adherence \\ 2 March 2013 \\ Number of times this article has been viewed
}

\author{
Amado Rivero-Santana' \\ Lilisbeth Perestelo-Perez ${ }^{2,3}$ \\ Jeanette Pérez-Ramos' \\ Pedro Serrano-Aguilar ${ }^{2,3}$ \\ Carlos De las Cuevas ${ }^{2,4}$ \\ 'Canary Islands Foundation of \\ Health and Research, ${ }^{2}$ Red de \\ Investigacion en Servicios de \\ Salud en Enfermedades Cronicas \\ (REDISSEC), Santa Cruz de Tenerife, \\ ${ }^{3}$ Evaluation Unit, Canary Islands \\ Health Service, Santa Cruz de \\ Tenerife, ${ }^{4}$ Department of Psychiatry, \\ University of La Laguna, Canary \\ Islands, Spain
}

Background: The literature shows that compliance with antidepressant treatment is unsatisfactory. Several personal and disease-related variables have been shown to be related to compliance behavior. The objective of this study was to review the literature about sociodemographic and clinical predictors of compliance in patients with depressive disorders.

Methods: The Medline, Embase, Cochrane Central, PsycInfo, and Cinahl databases were searched until May 2012. Studies that analyzed sociodemographic and clinical predictors or correlates of compliance in patients with depressive disorder were included. A quantitative synthesis was not performed because of the heterogeneity and availability of the data reported. For similar reasons, the results were not classified according to the different phases of treatment. The search was limited to studies published in English and Spanish.

Results: Thirty-two studies fulfilled the inclusion criteria. The most consistent associations with compliance were found for age (older patients showed more compliance) and race (white patients were more likely to adhere to treatment than minority ethnic groups). Few studies assessed clinical factors, and the most plausible predictors of compliance were certain comorbidities and substance abuse. Severity of depression did not play an important role in predicting compliance.

Conclusion: The impact of the variables studied on compliance behavior appeared to be inconsistent. Identifying potential predictors of compliance with antidepressant treatment is important, both for the routine practice of the mental health professional and for refining interventions to enhance adherence and target them to specific populations at risk of noncompliance.

Keywords: adherence, antidepressants, compliance, depression, predictors

\section{Introduction}

Depressive disorders have become a priority public health concern because of their high prevalence and global disease burden, mainly as a result of the disability caused. The total number of people with depression in Europe reached 21 million in the year 2004, ${ }^{1}$ and the World Health Organization estimates that, by the year 2020, depression will become the second most important cause of disability worldwide. ${ }^{2}$ Despite the availability of effective drugs for the treatment of depression, a significant percentage of patients do not achieve full remission of symptoms. ${ }^{3}$ Furthermore, approximately $50 \%$ of patients experience recurrence, and the probability of another depressive episode increases with each case of recurrence. ${ }^{4}$ Therefore, for many patients, depression presents as a chronic disorder that requires lifelong antidepressant treatment to
Correspondence: Amado Rivero-Santana Canary Islands Foundation of Health and Research, Evaluation Unit of the Canary Islands Health Service, C/Perez de Rozas 5, $4^{\text {a }}$ Planta, Santa Cruz de Tenerife 38004, Spain

Tel +34922475755

Fax +34922 475768

Email amado.riverosantana@sescs.es 
prevent recurrences. For these reasons, most national guidelines recommend continuing treatment for 4-9 months after the current episode has remitted. ${ }^{5}$

In this context, compliance with antidepressant treatment becomes a crucial factor in order to reach the desired outcomes of treatment. Compliance has been defined as the extent to which a person's behavior, in terms of taking medication, following diets, or executing lifestyle changes, coincides with medical or health advice. ${ }^{6}$ It has been discussed whether the term "adherence" reflects a less paternalistic relationship towards patients than "compliance", or even if both terms should be replaced by concepts such as alliance or concordance, which implicitly represent a more patientcentered approach. ${ }^{7}$ This discussion is beyond the scope of this article, and therefore the terms "compliance" and "adherence" will be considered synonymous. It is accepted that nonadherence may refer to several distinct aspects of medication-taking behaviors, ie, failure to attend an initial appointment, failure to have the prescription filled, having the prescription filled but failing to take the medication, not following the frequency or dose instructions of the prescription, errors of purpose, or use of inadvertent combinations. ${ }^{8}$ For the purposes of this work, we will refer to "nonadherence" as partial compliance (missed doses) and to "discontinuation" as definitely discontinuing the medication.

It has been argued that if guideline recommendations about antidepressant treatment were followed exactly, the overall burden of depression (measured by disability-adjusted lifeyears) could be reduced by approximately $28 \% .{ }^{9}$ However, several clinical studies have shown that patient adherence with antidepressants is quite unsatisfactory, specifically with regard to long-term maintenance treatment. Up to $42 \%$ of patients discontinue treatment after 12 weeks, ${ }^{5}$ and partial compliance has been estimated to be $45 \% .{ }^{10}$ Reasons for noncompliance include a wide range of factors, related to patient or treatment characteristics, as well as to patient-physician interaction. ${ }^{11,12}$ Adverse effects of medications have been shown to play an important role in treatment discontinuation and adherence, although the introduction of new-generation antidepressants with fewer side effects has, to some extent, overcome this problem. Other variables that have been related to nonadherence are perceived lack of efficacy, poor instructions, lack of information about the condition and its treatment, "polyprescribing", or a difficult dosing regimen. ${ }^{13}$ In the case of mental disorders, factors related to the effect of the illness, such as lack of awareness of the disease and depressed mood or cognitive impairment, may act as additional barriers for adequate adherence to treatment.
One of the more important difficulties in research on adherence is its measurement. Several methods have been used, including patient self-report, physician rating, pill count, prescription fills count, drug/metabolite plasma concentration, or the Medication Event Monitoring System. Patient and physician reports are subject to reliability problems, while the other techniques are expensive or not acceptable to all patients because of their invasiveness. Studies that have analyzed the concordance between these different methods of assessing adherence have reported acceptable correlations between them. ${ }^{14-16}$

As part of a wider review concerning predictors of compliance with antidepressant medications, the aim of this study was to review the literature on sociodemographic and clinical (disease-related) predictors or correlates of compliance with antidepressants in patients with depressive disorders.

\section{Materials and methods}

The Medline, Embase, Cochrane Central, PsycInfo, and Cinahl databases were searched from January 1990 to May 2012, and two of the authors independently selected all relevant English and Spanish language publications. References were first selected by title and abstract and then obtained in full text. In addition to this search strategy, references lists were searched manually. Once the selection process was finished, discrepancies between authors were discussed and resolved by consensus.

To be included in the review, studies had to be observational and include adult and/or elderly patients diagnosed with a depressive disorder by a health care provider or the researchers of the study. They had to analyze, either by retrospective, cross-sectional, or prospective design, some of the following predictors or correlates of compliance with antidepressant medications: age, gender, race, education, living situation/marital status, income, employment status, diagnosis subtype, severity of depression, previous episodes, psychiatric and medical comorbidities, cognitive impairment, and perceived health or health-related quality of life. Studies were excluded if they included patients with bipolar or psychotic disorders, if they only included patients with depression along with a medical illness, or if participants were taking antidepressants but the diagnosis was not reported (or only assessed by participant self-report).

Data on the study sample, design, predictor variables, follow-up duration, definition and method of assessing compliance, and statistical methods were extracted. Results of multivariate analyses will be commented on, unless only bivariate analyses were reported in the study. We classified 
the results into two types of noncompliance behavior, ie, discontinuation (stopping taking the medication) and nonadherence (intermittent or partial compliance).

\section{Results}

After eliminating duplicates, 1690 references were obtained. One hundred and twenty were selected by title/abstract, and 34 were finally included, comprising 32 studies (one study was reported in two references, ${ }^{17,18}$ and another ${ }^{19}$ used a subsample of the parent study ${ }^{20}$ ). Twenty-one additional references obtained by hand searching were examined, but none fulfilled the inclusion criteria. Among the excluded studies that assessed predictors or correlates of compliance, the most common reason for exclusion was that a formal diagnosis of depression was not required, but only the use of antidepressants.

Table 1 shows the characteristics of the studies included. Twenty-three studies were performed in the United States, ${ }^{20-42}$ two in Canada, ${ }^{43,44}$ two in Spain, ${ }^{45,46}$ two in Taiwan, ${ }^{47,48}$ one in the United Kingdom, ${ }^{49}$ one in Belgium, ${ }^{17,18}$ and one in New Zealand. ${ }^{50}$ Sample sizes were less than 100 in eight studies, 100-600 in eleven studies, and more than 2000 in 12 studies. Given that the two studies ${ }^{29,30}$ with the smallest sample sizes (30 and 22 subjects) did not yield statistically significant results for any of the variables analyzed, they are not considered in the counting of results, in order to limit the exposition to a group of studies with acceptable statistical power. Tables 2 and 3 show the results obtained.

\section{Predictors of treatment discontinuation}

Seven studies offered data about treatment discontinuation. Three used a retrospective design, ${ }^{36,37,42}$ one was a crosssectional study, ${ }^{24}$ and three used a prospective design. ${ }^{17,18,41,47}$ To assess discontinuation, four studies used self-report measures, ${ }^{17,18,24,36,41}$ two used prescription fills data, ${ }^{37,42}$ and one considered discontinuation as not attending follow-up visits. ${ }^{47}$ Two studies assessed discontinuation at 3 months, ${ }^{36,41}$ three at 6 months, ${ }^{17,18,37,47}$ and one at 12 months. ${ }^{42}$

\section{Sociodemographic predictors}

Potential sociodemographic predictors investigated were age, gender, race, educational level, living situation/marital status, income, and employment status. All studies assessed the effect of age on treatment discontinuation, and three of them obtained significant results. ${ }^{37,41,42}$ Older age was associated with both lower rates ${ }^{41}$ and a longer time ${ }^{42}$ to discontinuation. Sanglier et al found an interaction between age and antidepressant dispensing year, in that before 2006 , older adults were more likely to discontinue than younger ones, but the opposite was found after that year. ${ }^{37}$

Six studies offered data about gender differences for dis-

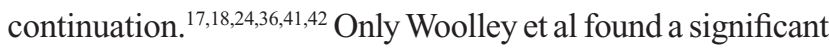
effect, with men being significantly more likely to discontinue with treatment. ${ }^{41}$ Demyttenaere et al found an interaction between gender and functional improvement, in that the risk of discontinuation for women related to improvement in family functioning, while in men it was related to improvements in occupational, social, and family functioning. ${ }^{18}$

Four studies analyzed the effect of race..$^{24,36,41,42}$ Olfson et al found that Hispanic patients (but not black or other minorities) showed higher discontinuation rates than white patients. ${ }^{36}$ Further, $\mathrm{Wu}$ et al reported a longer time to discontinuation in Caucasians versus African-American patients. ${ }^{42}$

Four studies assessed the effect of educational level on discontinuation, ${ }^{24,36,41,47}$ and only Olfson et al found a significant result, ie, individuals with 12 years of education or less showed higher rates of discontinuation than those with more than 12 years. ${ }^{36}$

Bull et al found that separate or divorced patients and those whose spouses had died discontinued significantly more often than married patients. ${ }^{24}$ The remaining two studies that assessed living situation and/or marital status of the participants did not obtain statistically significant results. ${ }^{36,41}$

Only Olfson et al assessed income status and found that patients with low incomes discontinued significantly more often than those with high incomes. ${ }^{36}$ None of the four studies that included employment status as a potential predictor of discontinuation found significant results. . $^{2,36,41,47}$

\section{Clinical predictors}

Potential clinical predictors investigated were diagnostic subtype, severity of depression, previous episodes, comorbidities, cognitive impairment, and perceived health status/ health-related quality of life. Hung et al found that individuals with chronic depression showed lower rates of discontinuation than those without the condition. ${ }^{47}$ The three studies that assessed severity did not find statistically significant associations with discontinuation of treatment. ${ }^{24,41,47}$ Bull et al found that it was improvement, and not level of depression, that predicted an adequate treatment duration. ${ }^{24}$ Only Sanglier et al examined the role of history of previous depressive episodes and did not find statistically significant associations with discontinuation. ${ }^{37}$ The two studies that assessed psychiatric and medical comorbidities did not find statistically significant associations. ${ }^{42,47}$ Olfson et al is the only study that assessed 


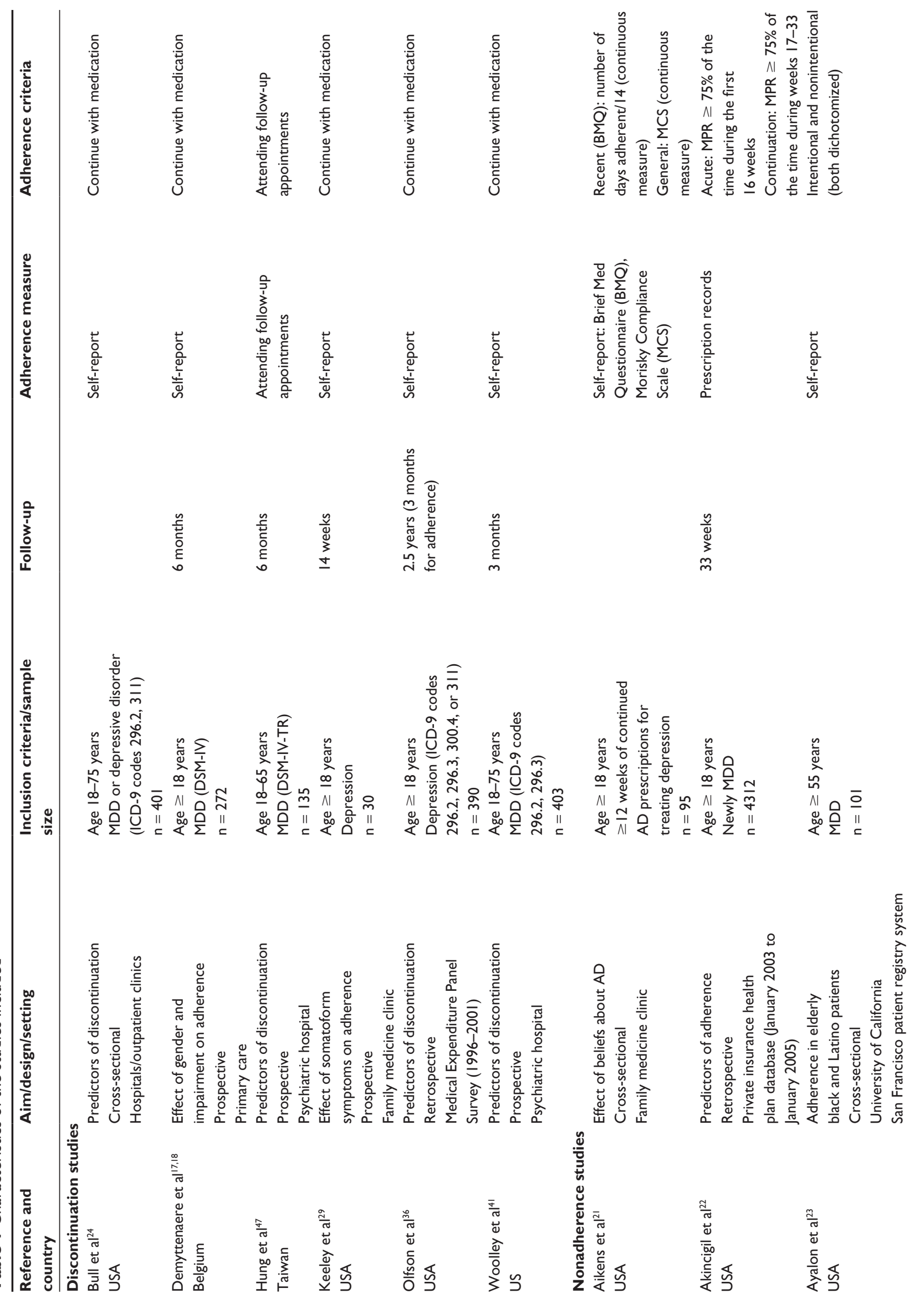



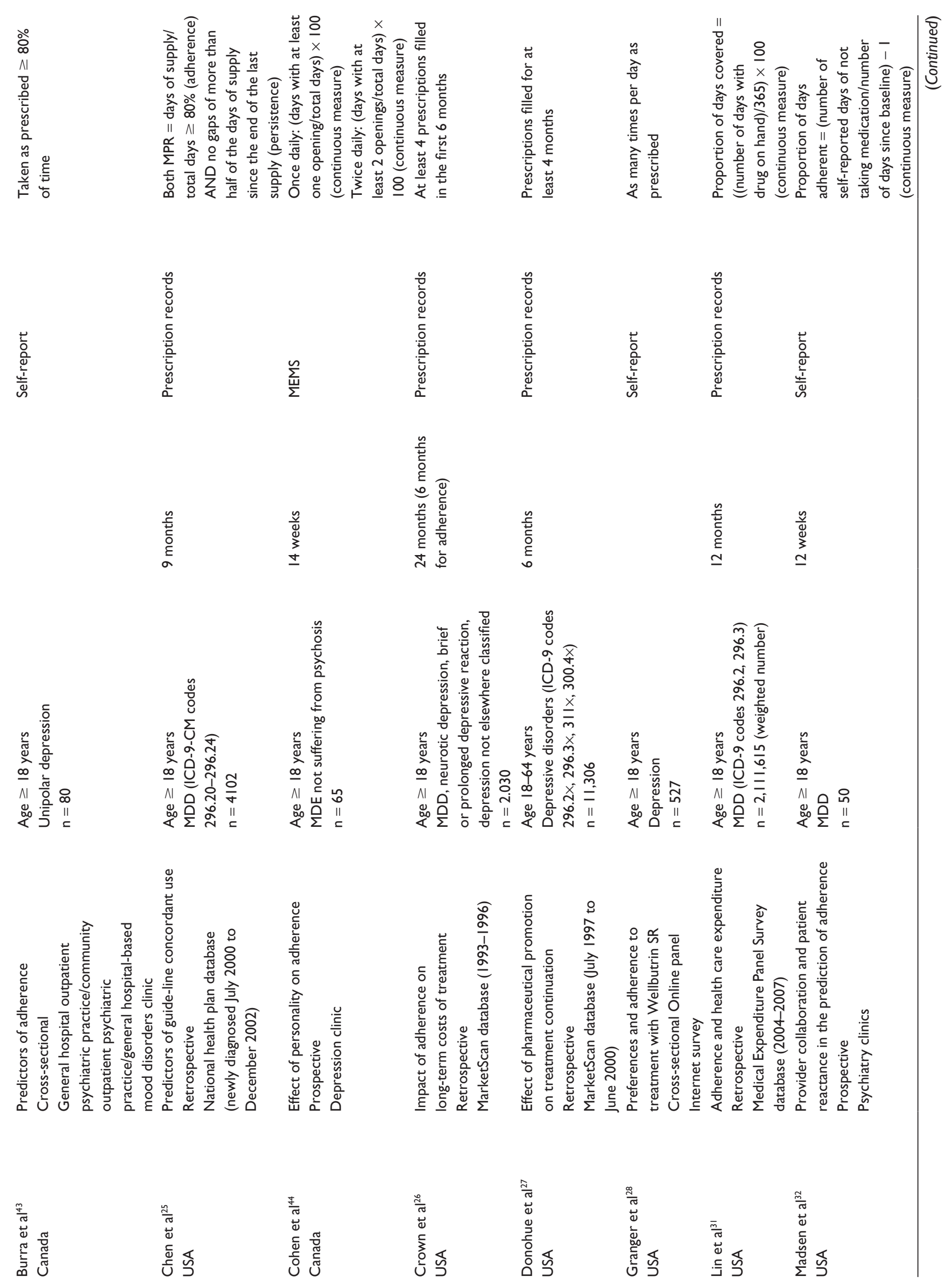


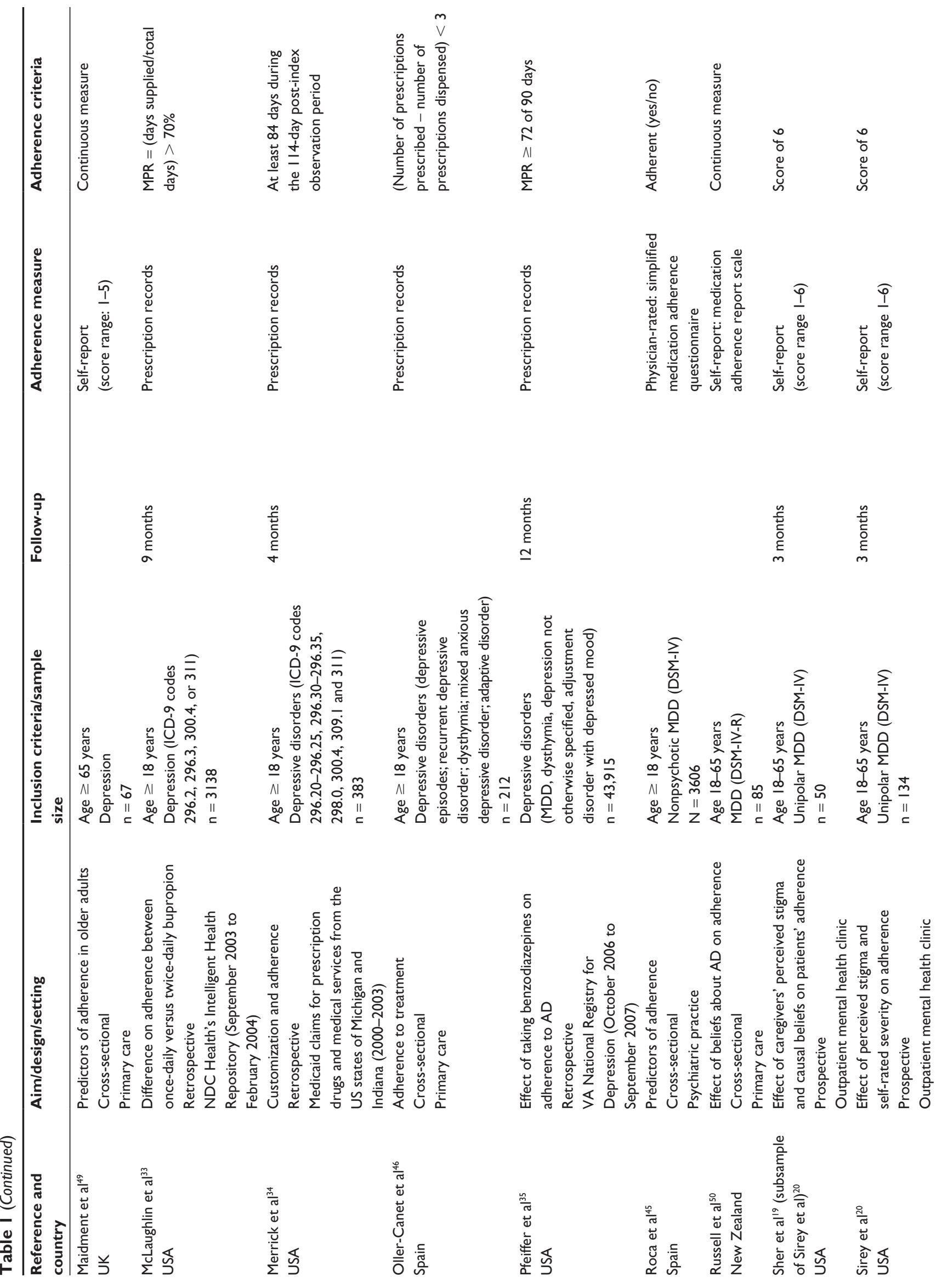




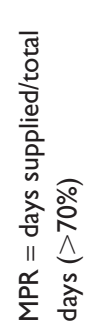
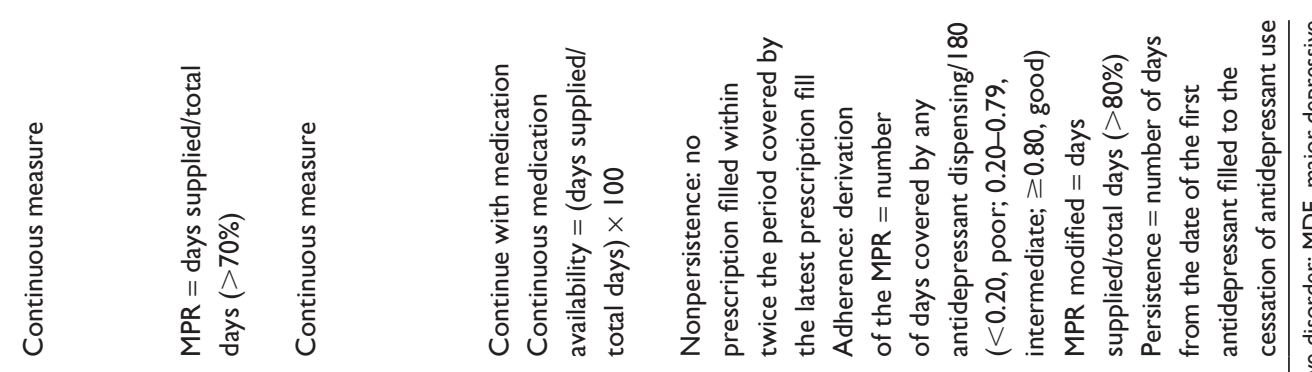

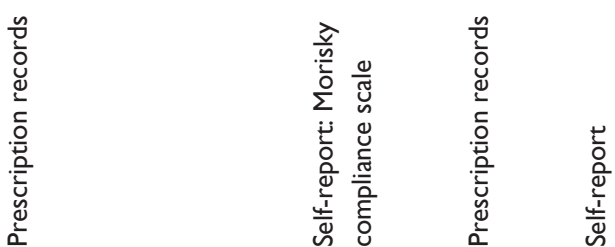

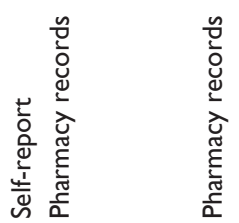

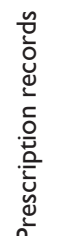

$\frac{n}{E}$
$\stackrel{5}{0}$
$\stackrel{0}{0}$

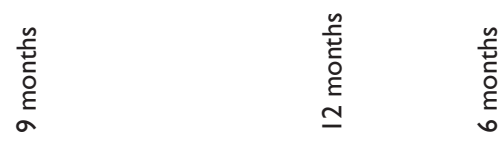

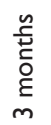

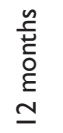
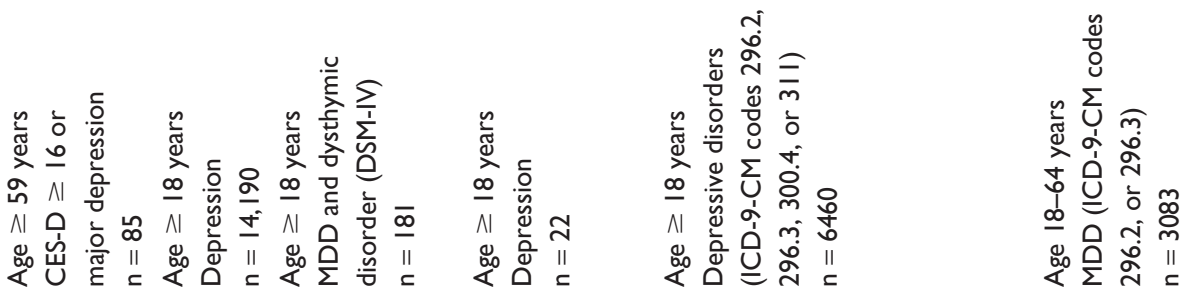

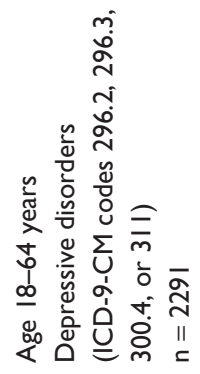

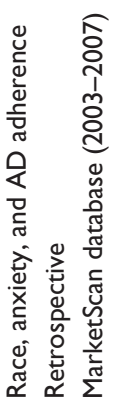

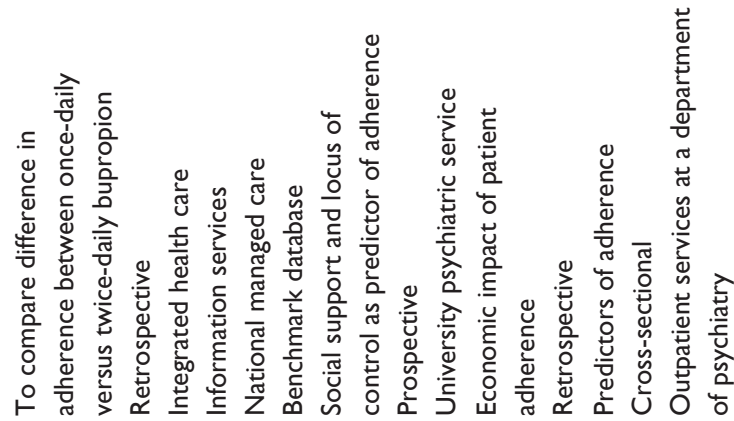

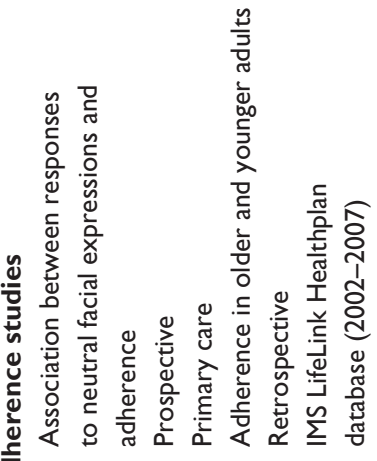

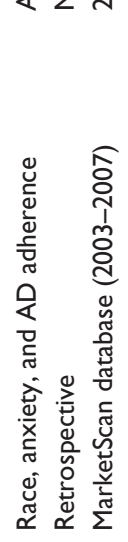

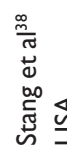

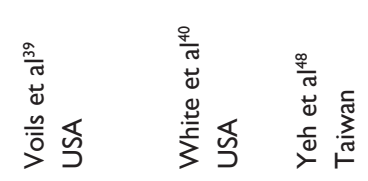

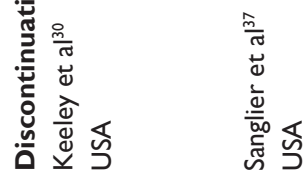

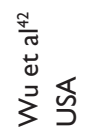


Table 2 Results of studies included (sociodemographic predictors)

\begin{tabular}{|c|c|c|c|}
\hline & Age & Gender & Race \\
\hline \multicolumn{4}{|l|}{ Discontinuation } \\
\hline $\begin{array}{l}\text { Bull et } \mathrm{al}^{24} \\
\text { USA }\end{array}$ & NS & NS & NS \\
\hline Demyttenaere et al ${ }^{17,18}$ & NS & NS & \\
\hline Belgium & & $\begin{array}{l}\text { Significant interaction with type of } \\
\text { impairment }\end{array}$ & \\
\hline Hung et $\mathrm{al}^{47}$ & NS & NS & \\
\hline Taiwan & & & \\
\hline $\begin{array}{l}\text { Keeley et a }{ }^{29} \\
\text { USA }\end{array}$ & NS & NS & NS \\
\hline $\begin{array}{l}\text { Olfson et } \text { al }^{36} \\
\text { USA }\end{array}$ & NS & NS & $\begin{array}{l}\text { Hispanic patients continue less than } \\
\text { white (OR } 0.58 ; 95 \% \mathrm{Cl} 0.36-0.94 \text { ) }\end{array}$ \\
\hline $\begin{array}{l}\text { Woolley et al }{ }^{41} \\
\text { USA }\end{array}$ & $\begin{array}{l}\text { Increases in age relate to less } \\
\text { discontinuation (OR } 0.98 ; 95 \% \mathrm{Cl} \\
0.96-1.00 \text { ) }\end{array}$ & $\begin{array}{l}\text { Males discontinue more (OR 2.02; } \\
95 \% \mathrm{Cl} \mathrm{I.16-3.49)}\end{array}$ & NS \\
\hline $\begin{array}{l}\text { Keeley et a }{ }^{10} \\
\text { USA }\end{array}$ & NS & NS & NS \\
\hline $\begin{array}{l}\text { Sanglier et al }{ }^{37} \\
\text { France }\end{array}$ & Interaction with dispensing year & & \\
\hline $\begin{array}{l}\text { Wu et al }{ }^{42} \\
\text { USA }\end{array}$ & $\begin{array}{l}\text { Age } 5 \mathrm{I}-60 \text { years more persistent than } \\
\text { those aged I8-30 years (HR 0.6I; } 95 \% \\
\mathrm{Cl} 0.5 \mathrm{I}-0.74 \text { ) }\end{array}$ & NS & $\begin{array}{l}\text { African-Americans less persistent } \\
\text { than Caucasians (HR I.47; } 95 \% \mathrm{Cl} \\
\text { I.30-I.65) }\end{array}$ \\
\hline Nonadherence & & & \\
\hline $\begin{array}{l}\text { Aikens et } \mathrm{al}^{21} \\
\text { USA }\end{array}$ & NS & NS & \\
\hline $\begin{array}{l}\text { Akincigil et } \mathrm{al}^{22} \\
\text { USA }\end{array}$ & $\begin{array}{l}\text { Acute phase: Ages } 40-49 \text { years (OR } \\
\text { I.7I; } 95 \% \mathrm{Cl} \text { I.36-2.I5), 50-64 years } \\
\text { (OR 2.48; } 95 \% \mathrm{Cl} \text { I.94-3.I5), and } \geq \\
65 \text { years (OR I.96; } 95 \% \mathrm{Cl} \text { I.34-2.85) } \\
\text { more adherent than I8-25 years }\end{array}$ & NS & \\
\hline $\begin{array}{l}\text { Ayalon et } \mathrm{al}^{23} \\
\text { USA }\end{array}$ & NS & & NS \\
\hline $\begin{array}{l}\text { Burra et a } \mathrm{l}^{43} \\
\text { Canada }\end{array}$ & NS & $\begin{array}{l}\text { Females are less nonadherent than } \\
\text { males (OR 5.I2; 95\% Cl I.09-24.I) }\end{array}$ & \\
\hline $\begin{array}{l}\text { Chen et } \mathrm{a}^{25} \\
\text { USA }\end{array}$ & $\begin{array}{l}\text { Acute phase: age } 35-49 \text { years (OR I.38; } \\
95 \% \mathrm{Cl} \text { I. } 19-1.60 \text { ), 50-64 years (OR } \\
\text { I.39; } 95 \% \mathrm{Cl} \text { I.15-I.68), and } \geq 65 \text { years } \\
\text { (OR } 2.77 ; 95 \% \mathrm{Cl} \text { I.67-4.58) more } \\
\text { adherent than I8-34 years } \\
\text { Continuation phase: } \\
\text { age } 35-49 \text { years (OR I.40; } 95 \% \mathrm{Cl} \\
\text { I.I2-I.74) and 50-64 years (OR I.8I; } \\
95 \% \mathrm{Cl} \text { I.36-2.39), more adherent than } \\
\text { I8-34 years }\end{array}$ & NS & \\
\hline Cohen et $\mathrm{al}^{44}$ & NS & NS & \\
\hline Canada & & & \\
\hline $\begin{array}{l}\text { Crown et a }{ }^{26} \\
\text { USA }\end{array}$ & $\begin{array}{l}\text { Increases in age relate to better } \\
\text { adherence }(\mathrm{t}=2.868 ; P<0.01)\end{array}$ & $\begin{array}{l}\text { Females adhere more than males } \\
(\mathrm{t}=2.83 \mathrm{I} ; \mathrm{P}<0.0 \mathrm{I})\end{array}$ & \\
\hline
\end{tabular}


Education

NS

NS

Those with I2 (OR $0.53 ; 95 \% \mathrm{Cl}$

$0.35-0.79$ ) or less (OR $0.64 ; 95 \% \mathrm{Cl}$

$0.42-0.92$ ) years of education continue less than those with $>12$ years

NS

NS

NS

NS

Those who had not completed NS

Separated, divorced, or widowed subjects discontinued more than married people (OR 2.83; 95\% Cl I.49-5.39)

NS

NS

Those with low incomes continue less than those with high incomes (OR 0.64; 95\% Cl 0.4I-0.99)

NS

NS

Acute phase: those earning $\$ 50,000-70,000$

(OR I.22; 95\% Cl I.05-I.42) and $\geq \$ 70,000$

(OR I.30; 95\% Cl I.II-I.53) adhere more than those under $\$ 50,000$

Continuation phase: those earning $\$ 50,000$

70,000 (OR I.25; 95\% Cl I.002-I.55) adhere more than those under $\$ 50,000$

NS 
Table 2 (Continued)

\begin{tabular}{|c|c|c|c|}
\hline & Age & Gender & Race \\
\hline $\begin{array}{l}\text { Donohue et } \mathrm{al}^{27} \\
\text { USA }\end{array}$ & $\begin{array}{l}\text { Older patients more adherent (data not } \\
\text { reported) }\end{array}$ & $\begin{array}{l}\text { Women more adherent than men } \\
\text { (data not reported) }\end{array}$ & \\
\hline Granger et $\mathrm{al}^{28}$ & Likelihood & Females were nearly twice as & \\
\hline USA & $\begin{array}{l}\text { of nonadherence decreased with age } \\
\text { (data not reported) }\end{array}$ & $\begin{array}{l}\text { likely as males to be nonadherent } \\
\text { (data not reported) }\end{array}$ & \\
\hline $\begin{array}{l}\text { Lin et } \mathrm{al}^{31} \\
\text { USA }\end{array}$ & NS & NS & $\begin{array}{l}\text { Hispanics less adherent than non- } \\
\text { Hispanic whites }(P<0.05) \text { and } \\
\text { other ethnicities }(P<0.0 \text { I })\end{array}$ \\
\hline $\begin{array}{l}\text { Maidment et al }{ }^{49} \\
\text { UK }\end{array}$ & NS & NS & \\
\hline $\begin{array}{l}\text { McLaughlin et } \mathrm{al}^{33} \\
\text { USA }\end{array}$ & $\begin{array}{l}\text { Increased age relate to better } \\
\text { adherence (OR I.0I; } 95 \% \text { Wald } \mathrm{Cl} \\
\mathrm{I} .008-1.012)\end{array}$ & NS & \\
\hline $\begin{array}{l}\text { Merrick et } \mathrm{al}^{34} \\
\text { USA }\end{array}$ & $\begin{array}{l}\text { Age } 60-74 \text { years (OR } 2.4 ; 95 \% \mathrm{Cl} \\
\text { I.2-4.8) and } \geq 75 \text { years (OR } 2.7 ; 95 \% \\
\mathrm{Cl} \text { I.4-5.4) more adherent than } 45-59 \\
\text { years }\end{array}$ & NS & $\begin{array}{l}\text { Whites more adherent than } \\
\text { nonwhites (OR 2.4; 95\% Cl I.3-4.3) }\end{array}$ \\
\hline $\begin{array}{l}\text { Oller-Canet et } \mathrm{al}^{46} \\
\text { Spain }\end{array}$ & NS & $\begin{array}{l}\text { NS } \\
\text { Significant interaction with type of } \\
\text { AD (among SSRI users, women were } \\
\text { more adherent than men, } \\
P=0.015)\end{array}$ & \\
\hline $\begin{array}{l}\text { Pfeiffer et } \mathrm{al}^{35} \\
\text { USA }\end{array}$ & $\begin{array}{l}\text { Increased age related to better } \\
\text { adherence (OR I.0I; } 95 \% \mathrm{Cl} \text { I.0I-I.0I) }\end{array}$ & $\begin{array}{l}\text { Men show less adherence (OR 0.88; } \\
95 \% \mathrm{Cl} 0.83-0.94)\end{array}$ & $\begin{array}{l}\text { Blacks (OR 0.47; } 95 \% \mathrm{Cl} 0.44-0.50) \\
\text { and others (OR 0.82; } 95 \% \mathrm{Cl} \\
0.72-0.93 \text { ) less adherent than } \\
\text { whites. Hispanics less adherent than } \\
\text { non-Hispanics (OR } 0.66 ; 95 \% \mathrm{Cl} \\
0.70-0.72 \text { ) }\end{array}$ \\
\hline $\begin{array}{l}\text { Roca et } \text { al }^{45} \\
\text { Spain }\end{array}$ & & $\begin{array}{l}\text { Male gender related to poor } \\
\text { adherence (data not reported) }\end{array}$ & \\
\hline $\begin{array}{l}\text { Sher et al }{ }^{19} \\
\text { (subsample of Sirey et al) }^{20} \\
\text { USA }\end{array}$ & NS & NS & \\
\hline $\begin{array}{l}\text { Sirey et } \mathrm{al}^{20} \\
\text { USA }\end{array}$ & $\begin{array}{l}\text { Age } \geq 60 \text { years better adherence than } \\
<60 \text { years (OR } 2.91 ; 95 \% \mathrm{Cl} \text { I.03-8.24) }\end{array}$ & NS & NS \\
\hline $\begin{array}{l}\text { Stang et } \mathrm{al}^{38} \\
\text { USA }\end{array}$ & $\begin{array}{l}\text { Increased age related to better adherence } \\
\text { (OR I.026; } 95 \% \mathrm{Cl} \text { I.017-I.034) }\end{array}$ & NS & \\
\hline $\begin{array}{l}\text { Voils et al }{ }^{39} \\
\text { USA }\end{array}$ & NS & NS & \\
\hline $\begin{array}{l}\text { White et } \mathrm{al}^{40} \\
\text { USA }\end{array}$ & $\begin{array}{l}\text { Higher rate of patients }<40 \text { years in } \\
\text { nonadherent group }(P<0.001)\end{array}$ & NS & \\
\hline $\begin{array}{l}\text { Yeh et } \text { al }^{48} \\
\text { Taiwan }\end{array}$ & NS & NS & \\
\hline $\begin{array}{l}\text { Keeley et } \mathrm{al}^{30} \\
\text { USA }\end{array}$ & NS & NS & NS \\
\hline $\begin{array}{l}\text { Sanglier et } \mathrm{al}^{37} \\
\text { USA }\end{array}$ & Interaction with dispensing year & & \\
\hline $\begin{array}{l}\text { Wu et } \mathrm{al}^{42} \\
\text { USA }\end{array}$ & $\begin{array}{l}\text { Ages } 3 \mathrm{I}-40 \text { years (OR I.39; } 95 \% \mathrm{Cl} \\
\text { I.I5-I.67), } 4 \mathrm{I}-50 \text { years (OR I.73; } 95 \% \\
\mathrm{CI} \text { I.40-2.I4), 5I-60 years (OR I.90; } \\
95 \% \mathrm{CI} \text { I.45-2.49) and 6I-64 years } \\
\text { (OR I.9I; } 95 \% \mathrm{CI} \text { I.05-3.46) more } \\
\text { adherent than I8-30 years }\end{array}$ & NS & $\begin{array}{l}\text { African-American less adherent } \\
\text { than Caucasians (OR 0.60; } 95 \% \mathrm{Cl} \\
0.5 \mathrm{I}-0.72 \text { ) }\end{array}$ \\
\hline
\end{tabular}

Abbreviations: $\mathrm{Cl}$, confidence interval; NS, no significant results; HR, hazard ratio; OR, odds ratio; SSRI, selective serotonin reuptake inhibitor. 


Education

Living situation/marital status

NS

NS

NS

Lower educational level related to poor adherence (data not reported)

NS

NS

NS

NS
Living alone related to poor adherence (data not reported)

NS

NS

NS
Income

Employment

NS

NS

NS

Being unemployed related to poor adherence (data not reported)

NS
Higher income relates to worse adherence $(\mathrm{t}=-3.054 ; P<0.01)$
NS

NS 
Table 3 Results of the studies included (clinical predictors)

\begin{tabular}{|c|c|c|c|}
\hline & Diagnostic subtype & Severity & Previous episodes \\
\hline \multicolumn{4}{|c|}{ Discontinuation } \\
\hline Bull et $\mathrm{a}^{24}$ & & NSD in BDI-FS at 3 months & \\
\hline USA & & $\begin{array}{l}\text { Those improved at } 3 \text { months discontinue less } \\
\text { (OR } 0.40 ; 95 \% \mathrm{Cl} 0.20-0.82 \text { ) }\end{array}$ & \\
\hline \multicolumn{4}{|c|}{ Demyttenaere et al ${ }^{17,18}$} \\
\hline \multicolumn{4}{|c|}{ Belgium } \\
\hline Hung et $\mathrm{a}^{47}$ & Those with chronic depression & NS (HAM-D) & \\
\hline Taiwan & $\begin{array}{l}\text { discontinue less than those without } \\
(\mathrm{OR} 0.40,95 \% \mathrm{Cl} 0.20-0.8 \mathrm{I})\end{array}$ & & \\
\hline Keeley et $\mathrm{al}^{29}$ & & NS (PAI depression subscale) & \\
\hline \multicolumn{4}{|l|}{ USA } \\
\hline \multicolumn{4}{|l|}{ Olfson et $\mathrm{al}^{36}$} \\
\hline \multicolumn{4}{|l|}{ USA } \\
\hline Woolley et $\mathrm{al}^{41}$ & & NS (BDI) & \\
\hline \multicolumn{4}{|l|}{ USA } \\
\hline Keeley et $\mathrm{al}^{30}$ & NS & NS & \\
\hline \multicolumn{4}{|l|}{ USA } \\
\hline Sanglier et $\mathrm{a}^{37}$ & & & NS \\
\hline \multicolumn{4}{|l|}{ USA } \\
\hline \multicolumn{4}{|l|}{ Wu et $\mathrm{al}^{42}$} \\
\hline \multicolumn{4}{|l|}{ USA } \\
\hline \multicolumn{4}{|l|}{ Nonadherence } \\
\hline Aikens et $\mathrm{al}^{21}$ & & NS (PHQ-9) & \\
\hline \multicolumn{4}{|l|}{ USA } \\
\hline \multicolumn{4}{|l|}{ Akincigil et $\mathrm{al}^{22}$} \\
\hline \multicolumn{4}{|l|}{ USA } \\
\hline Ayalon et $\mathrm{al}^{23}$ & & NS (GDS) & \\
\hline \multicolumn{4}{|l|}{ USA } \\
\hline Burra et $a^{43}$ & & NS & \\
\hline \multicolumn{4}{|l|}{ Canada } \\
\hline \multicolumn{4}{|l|}{ Chen et $\mathrm{a}^{25}$} \\
\hline \multicolumn{4}{|l|}{ USA } \\
\hline Cohen et $\mathrm{al}^{44}$ & NS & NS (HDRS-I7) & NS \\
\hline \multicolumn{4}{|l|}{ Canada } \\
\hline Crown et $\mathrm{a}^{26}$ & MDD single episode & & \\
\hline \multirow[t]{4}{*}{ USA } & $(\mathrm{t}=-2.228 ; P<0.0 \mathrm{I})$, MDD recurrent & & \\
\hline & episode $(\mathrm{t}=-2.68 \mathrm{I} ; P<0.05)$ and & & \\
\hline & neurotic depression $(\mathrm{t}=-2.284$ & & \\
\hline & $P<0.0$ I) relate to worse adherence & & \\
\hline Donohue et $\mathrm{al}^{27}$ & NS & & NS \\
\hline \multicolumn{4}{|l|}{ USA } \\
\hline \multicolumn{4}{|l|}{ Lin et $\mathrm{al}^{31}$} \\
\hline USA & & & \\
\hline
\end{tabular}

USA 
NS (panic/agoraphobia, social phobia, specific phobia, PTSD, OCD, GAD, migraine)

\section{NS}

(number of chronic problems)

NS (anxiety disorders, medical comorbidities)

Acute phase: those with headache or migraine adhere less than those without (OR 0.82; 95\% Cl 0.67-0.99). Those with 2 or more CVD/diabetes conditions (OR 0.65; 95\% Cl 0.49-0.86) adhere less than those without. Those with alcohol (OR 0.49; $95 \%$ $\mathrm{Cl} 0.36-0.68$ ) or substance abuse (OR $0.72 ; 95 \%$ $\mathrm{Cl} 0.56-0.93)$ adhere less than those without these conditions

Acute phase:

NS (CDS, anxiety)

Those with substance abuse less adherent (OR 0.62; $95 \% \mathrm{Cl} 0.45-0.86$ ). Continuation phase: NS (anxiety, substance abuse) higher CDS relates to better adherence (OR I.13; 95\% Cl I.00-1.27)

Number of nonmental health illnesses relate to worse adherence $(t=-2.382 ; P<0.05)$
Those with fair or poor mental health status discontinue more at 3 months than those with excellent to good mental health status (OR I.96; $95 \% \mathrm{Cl}$ I.21-3.19)
Increases in cognitive impairment related to poor unintentional adherence (OR 0.43; 95\% Cl 0.20-0.89)
NS 
Table 3 (Continued)

\begin{tabular}{|c|c|c|c|}
\hline & Diagnostic subtype & Severity & Previous episodes \\
\hline Madsen et $\mathrm{al}^{32}$ & & NS (BDI-II) & \\
\hline \multicolumn{4}{|l|}{ USA } \\
\hline Maidment et $\mathrm{al}^{49}$ & & NS (GMSS-DS) & \\
\hline \multicolumn{4}{|l|}{ UK } \\
\hline \multicolumn{4}{|l|}{ McLaughlin et $\mathrm{al}^{33}$} \\
\hline \multicolumn{4}{|l|}{ USA } \\
\hline Merrick et $\mathrm{al}^{34}$ & NS & & \\
\hline \multicolumn{4}{|l|}{ USA } \\
\hline \multicolumn{4}{|l|}{ Oller-Canet et $\mathrm{al}^{46}$} \\
\hline \multicolumn{4}{|l|}{ Spain } \\
\hline \multicolumn{4}{|l|}{ Pfeiffer et $\mathrm{al}^{35}$} \\
\hline \multicolumn{4}{|l|}{ USA } \\
\hline Roca et $\mathrm{al}^{45}$ & & Nonadherent showed more severity (HDRS) & NS \\
\hline Spain & & $(\mathrm{t}=\mathrm{II} .3 ; P<0.00 \mathrm{I})$ & \\
\hline Russell et $\mathrm{al}^{50}$ & & Lower severity (BDI-II) relates to better & \\
\hline New Zealand & & adherence (Spearman rho 0.33; $P<0.001$ ) & \\
\hline Sirey et $\mathrm{al}^{20}$ & & NS (HAM-D) & \\
\hline \multicolumn{4}{|l|}{ USA } \\
\hline Sher et ll $^{19}$ & & NS (HAM-D) & NS \\
\hline \multicolumn{4}{|l|}{ (subsample of Sirey et al) $^{20}$} \\
\hline \multicolumn{4}{|l|}{ USA } \\
\hline \multicolumn{4}{|l|}{ Stang et $\mathrm{al}^{38}$} \\
\hline \multicolumn{4}{|l|}{ USA } \\
\hline Voils et $\mathrm{al}^{39}$ & & & NS \\
\hline \multicolumn{4}{|l|}{ USA } \\
\hline \multicolumn{4}{|l|}{ White et $\mathrm{al}^{40}$} \\
\hline \multicolumn{4}{|l|}{ USA } \\
\hline Yeh et $\mathrm{al}^{48}$ & & NS (BDI) & \\
\hline \multicolumn{4}{|l|}{ Taiwan } \\
\hline Keeley et $\mathrm{al}^{30}$ & NS & NS & \\
\hline \multicolumn{4}{|l|}{ USA } \\
\hline \multicolumn{4}{|l|}{ Wu et $\mathrm{al}^{42}$} \\
\hline USA & & & \\
\hline
\end{tabular}

Abbreviations: NS, no significant results; BDI-FS, Beck Depression Inventory (Fast Screening); CCl, Charlson Comorbidity Index; CDS, Chronic Disease Score; $\mathrm{Cl}$, confidence interval; COPD, chronic obstructive pulmonary disease; CVD, cardiovascular disease; GAD, generalized anxiety disorder; GDS, Geriatric Depression Scale; GMSS-DS, Geriatric Mental State Schedule-Depression Scale; HDRS, HAM-D Hamilton Depression Rating Scale; MDD, major depressive disorder; OCD, obsessivecompulsive disorder; OR, odds ratio; PAI, Personality Assessment Inventory; PHQ-9, Patient Health Questionnaire; PTSD, post-traumatic stress disorder.

cognitive limitations and did not obtain statistically significant results. ${ }^{36}$ Olfson et al found that participants with fair or poor mental health status discontinued more at 3 months than those with excellent or good status. ${ }^{36}$

\section{Predictors of treatment nonadherence}

Twenty-five studies assessed adherence with antidepressants. Twelve used retrospective designs, ${ }^{22,25-27,31,33-35,37,38,40,42}$ nine were cross-sectional studies, ${ }^{21,23,28,43,45,46,48-50}$ and four used prospective designs..$^{20,32,39,44}$ Ten studies used self-report measures, ${ }^{20,21,23,28,32,39,43,48-50} 13$ used prescription fills data, ${ }^{22,25,26,27,31,33-35,37,38,40,42,46}$ one used the Medication Event Monitoring System, ${ }^{44}$ and one used a physicianrated measure. ${ }^{45}$ Follow-up periods ranged between 3 and 12 months.

\section{Sociodemographic predictors}

Potential sociodemographic predictors investigated were age, gender, race, educational level, living situation/marital status, income, and employment status. All studies except 
Higher impairment related

to better adherence

(beta $0.102 ; P<0.05$ )

NS (CCl, nondepression behavioral health comorbidity)

NS (arterial hypertension, ischemic heart disease, diabetes mellitus, COPD, osteoporosis and dyslipidemia) PTSD (OR 0.95; 95\% Cl 0.90-0.99), and substance use disorder (OR 0.8I; 95\% Cl 0.77-0.85) worse adherence than not having these conditions. Other anxiety disorder (OR I.I0; 95\% Cl I.04-I.16) related to better adherence Medical comorbidities: higher rate in nonadherent $\left(\chi^{2}=15.9 ; P<0.001\right)$ Psychiatric comorbidities: NS

Nonadherence worse in mental $(P<0.001)$ and physical health $(P=0.001)$

Adherent group higher in CDS $(P<0.0001)$

NS (CDS)

Those with anxiety disorders more adherent than

those without (OR I.55; 95\% Cl I.27-1.90)

Those with 2 (OR I.30; $95 \% \mathrm{Cl} \mathrm{I.03-1.63)} \mathrm{or} \geq 3$

medical conditions (OR I.34; 95\% Cl I.06-1.69)

more adherent than those with no comorbidities

three ${ }^{32,45,50}$ offered data on age, and 13 found statistically significant associations. ${ }^{20,22,25-28,33-35,37,38,40,42}$ As in the case of discontinuation, Sanglier et al found an interaction between age and year of drug dispensing. ${ }^{37}$ In all the remaining studies, increasing age was associated with better adherence.

Only four studies did not assess gender as a potential predictor of adherence..$^{23,32,37,50}$ For the remaining ones, five obtained statistically significant differences favoring women, ${ }^{26,27,35,43,45}$ while in one study women were almost twice as likely as men to be nonadherent. ${ }^{28}$ Another study also found that women were more adherent than men, but only among users of selective serotonin uptake inhibitors. ${ }^{46}$

Six studies analyzed race..$^{20,23,31,34,35,42}$ Four of them showed that white patients were significantly more likely to adhere to treatment. ${ }^{31,34,35,42}$ Ayalon et al, who did not find statistically significant results, included only black and Hispanic patients. ${ }^{23}$ When more than two ethnic categories were compared, Hispanic patients showed the worst adherence rates. ${ }^{31,35}$

Two of nine studies obtained statistically significant results for educational level..$^{20,21,23,28,31,39,43,45,48}$ Burra et al found 
that patients who had not completed post-secondary education showed lower rates of adherence compared with those above that educational level. ${ }^{43}$ Roca et al found that those with a lower educational level showed poor adherence, but the article did not report how this variable was measured or the numeric results. ${ }^{45}$

Six studies analyzed living situation/marital status, ${ }^{20,39,43,45,48,49}$ and only Roca et al found a statistically significant association, ie, living alone was significantly related to poor adherence. ${ }^{45}$

Two of five studies found that income was significantly related to adherence, with opposite results..$^{20,22,23,31,48}$ Akincigil et al found that patients with an income level lower than $\$ 50,000$ had a lower rate of adherence compared with those above that level. ${ }^{22}$ Yeh et al found a statistically significant linear inverse relationship between income and adherence. ${ }^{48}$ Only Roca et al, ${ }^{45}$ of six studies, ${ }^{20,27,28,43,45,48}$ obtained a statistically significant effect for employment status, with unemployed participants showing lower adherence rates, and once again, descriptive data and statistical tests were not reported.

\section{Clinical predictors}

Potential clinical predictors investigated were diagnostic subtype, severity of depression, previous episodes, comorbidities, cognitive impairment, and perceived health status/ health-related quality of life. Four studies assessed diagnostic subtype, ${ }^{26,27,34,44}$ and only Crown et al found statistically significant associations, ie, major depression disorder and neurotic depression related independently to worse adherence. ${ }^{26}$

Of ten studies that assessed the influence of severity of depression, ${ }^{20,21,23,32,43,44,45,48-50}$ only Roca et al and Russell et al found that it was related to worse adherence. ${ }^{45,50}$ None of the five studies that assessed previous history of depressive episodes found a statistically significant relationship with adherence. $^{20,27,39,44,45}$

Ten studies assessed psychiatric and/or medical comorbidities, ${ }^{22,25,26,31,34,35,40,42,45,46}$ and seven found significant associations with adherence..$^{22,25,26,35,40,42,45}$ Regarding medical comorbidities, the direction of the significant relationship was inconsistent across studies; three of them obtained better adherence for patients with a higher number of comorbid diseases, ${ }^{25,40,42}$ while another three found the opposite result. ${ }^{22,26,45}$ Regarding psychiatric comorbidities, substance abuse was a significant predictor of nonadherence in the three studies that assessed this. ${ }^{22,25,35}$ Comorbid anxiety disorders (except post-traumatic stress disorder, which predicted worse adherence in Pfeiffer et al $)^{35}$ was significantly related to better adherence in two studies, ${ }^{35,42}$ but another three studies did not show statistically significant results. ${ }^{22,25,26,31}$

Cognitive limitations were assessed in two studies, with statistically significant results, but in opposite directions. Ayalon et al, in a sample of African-American and Hispanic patients, found that cognitive limitation was related to unintentional nonadherence, ${ }^{23}$ while Maidment et al found that higher impairment predicted better adherence. ${ }^{49}$

Only Roca et al, ${ }^{45}$ out of four studies, ${ }^{23,31,39,45}$ obtained a statistically significant relationship between perceived health status and/or health-related quality of life and adherence, with better mental and physical health in adherent patients.

\section{Discussion}

The results of this review show inconsistent associations between the predictors studied and compliance with antidepressants. Among the sociodemographic factors, age and race appear to be the variables more consistently related to adherence. Regarding age, only two studies found a statistically significant association between older age and a lower probability of (or a longer time to) discontinuation. However, in the cases of adherence (partial compliance), approximately $60 \%$ of the studies that assessed this found statistically significant relationships, showing in all cases a positive association between older age and adherence. This effect seemed to be more intense in those studies that measured age categorically than in those which included age as a continuous variable, suggesting a nonlinear association between both factors. Depression in older adults is less well understood and presents unique clinical challenges, including more comorbidities and prescribed medications, and therefore it has been argued that the complexity of their treatment regimens along with other characteristics of aging, such as cognitive impairment, might act as barriers to adequate compliance. ${ }^{51,52}$ Alternatively, it could be argued that the higher number of comorbidities and prescribed medications could make older people more accustomed to taking them, leading to better adherence. However, none of these explanations is supported by the results obtained in this review, because the better adherence observed in older people was independent of the effect of the number of comorbidities and/or medications taken. . $2,25,26,34,42$ On the other hand, cognitive impairment was a significant predictor of unintentional nonadherence in Ayalon et al, ${ }^{23}$ but the opposite was found in the study by Maidment et al, a finding that the authors explained by the more intense support that these impaired patients would receive from their caregivers. ${ }^{49}$ 
Regarding race, the results are consistent, with higher compliance by white patients, and Hispanic patients showing the poorest adherence. As has been pointed out in other medical conditions, these ethnic differences might be accounted for by different belief systems about the nature of the disease or the potential outcomes of available treatments, as well as less trust in the health system and its professionals. ${ }^{53,54}$ It has been shown that African-American and Hispanic patients, compared with white patients, find antidepressants less acceptable, and are less likely to believe that medications are effective and that depression is biologically based, and more likely to believe that antidepressants are addictive and that counseling and prayer are effective in treating depression. ${ }^{55,56}$

Low educational level has been considered to be a potential risk factor for poor adherence, because individuals with less education may have more difficulty in understanding treatment regimens, medical recommendations, or the nature of the disease from which they suffer. ${ }^{57,58}$ However, results of the studies included here suggest that this is not an important independent predictor of compliance with antidepressant treatment, although it could interact negatively with other variables, such as patient attitudes or beliefs about treatment. Regarding gender, the results indicate better adherence for women, but most studies did not find a statistically significant relationship. Nonetheless, it appears that the attribution of higher noncompliance in women as previously reported cannot be sustained. ${ }^{11,59}$ Only one study that assessed compliance with bupropion treatment obtained a significantly worse result for women. ${ }^{28}$

Among clinical factors, it seems clear that severity of depression by itself is not a significant predictor of compliance behavior. Only two cross-sectional studies obtained statistically significant findings, which could be explained by an inverse causal association, ie, that better adherence leads to reduced severity of the disease. The course of symptoms, rather than the severity level at any moment during treatment, is more likely to predict nonadherence, and several studies have shown that both improvement in symptoms and lack of treatment efficacy may be responsible for poor compliance. ${ }^{8,60,61}$

Medical comorbidities have been shown to relate significantly to both good and poor adherence in the studies included. As commented above, living with the experience of various diseases may provide patients with greater "expertise" in managing medications, but in interaction with other variables, such as cognitive impairment, low educational level, or incomplete or inadequate physician's instructions, it could also result in a complex regimen that hinders compliance. For the rest of the clinical variables analyzed, few studies were found that enabled us to ascertain their effect on compliance behavior.

This study aimed to identify nonmodifiable factors related to compliance with antidepressant therapy in patients with depressive disorders. An important limitation is the lack of a quantitative synthesis of results, but the fact that the studies included showed considerable heterogeneity in the statistical methods used and the predictors assessed prompted us to show the results in a narrative manner. Secondly, because predictors of compliance are not the same in different treatment phases, ${ }^{59}$ we could not classify the results according to the follow-up duration of the studies because most of them used periods longer than 3 months without differentiating between acute and continuation phases, and cross-sectional studies included patients with different time frames since the onset of treatment. Third, we have limited the analyses to observational studies because some authors have argued that compliance rates and risk factors could be different in naturalistic studies and clinical trials. ${ }^{59}$ Finally, the bibliographic search was restricted to studies in English or Spanish.

Appropriate prescription of antidepressant drugs is a core element in the delivery of modern mental health, with antidepressants widely used not only to relieve symptoms and cure conditions but to prevent relapses in the future. In the research on variables that influence compliance with antidepressant medications, sociodemographic and disease-related variables have received less attention than more modifiable factors, such as treatment characteristics (number and frequency of doses, type of drug, or quality of follow-up care) or patient attitudes, beliefs, and preferences concerning the disease and its treatment. However, we consider that identifying all potential predictors of compliance behavior is necessary to enrich existing theoretical models about compliance behavior, so that they can explain the complexity of this phenomenon better. To attain these aims, more research is needed on mediating factors that could account for the relationships between nonmodifiable variables, such as age or race, and adherence with antidepressants. Identifying predictors of compliance behavior might also be useful in the daily practice of mental health professionals, enabling them to detect potentially noncompliant patients more accurately, and consequently targeting specific interventions to patients or populations with distinct characteristics that could lead to treatment nonadherence. 


\section{Acknowledgments}

This work was supported by the Instituto de Salud Carlos III, FEDER Unión Europea (PI10/00955). We thank Leticia Cuellar, María del Carmen Bujalance, and Esperanza Ciruela, who kindly assisted the authors in the electronic bibliographic search and acquisition of the relevant papers.

\section{Disclosure}

The authors report no conflicts of interest in this work.

\section{References}

1. Sobocki P, Jonsson B, Angst J, Rehnberg C. Cost of depression in Europe. J Ment Health Policy Econ. 2006;9:87-98.

2. Murray CJL, López AD. The Global Burden of Disease, a Comprehensive Assessment of Mortality and Disability from Diseases, Injuries, and Risk Factors in 1990 and Projected to 2020. Harvard, MA: Harvard University Press; 1996.

3. National Collaborating Centre for Mental Health. Depression: The Treatment and Management of Depression in adults (Updated Edition). National Clinical Practice Guideline Number 90. London, UK: British Psychological Society and Royal College of Psychiatrists; 2009.

4. Solomon DA, Keller MB, Leon AC, et al. Multiple recurrences of major depressive disorder. Am J Psychiatry. 2000;157:229-233.

5. Karasu TB, Gelenberg A, Merriam A, et al. Practice Guideline for the Treatment of Patients with Major Depressive Disorder, 2nd ed. Washington, DC: American Psychiatric Association; 2000.

6. Haynes RB. Introduction. In: Haynes RB, Taylor WD, Sacket DL, editors. Compliance in Health Care. Baltimore, MD: Johns Hopkins University Press; 1979.

7. Horne R, Weinman J, Barber N, Elliott R, Morgan M, Cribb A. Concordance, adherence and compliance in medicine taking. Report for the National Coordinating Centre for NHS Service Delivery and Organisation R and D (NCCSDO). London, UK: National Coordinating Centre for NHS Service Delivery and Organisation Research and Development; 2005. Available from: http://www.medslearning.leeds. ac.uk/pages/documents/useful_docs/76-final-report\%5B1\%5D.pdf. Accessed February 5, 2013.

8. Demyttenaere K. Risk factors and predictors of compliance in depression. Eur Neuropsychopharmacol. 2003;13:S69-S75.

9. Vos T, Haby MM, Barendregt JJ, Kruijshaar M, Corry J, Andrews G. The burden of major depression avoidable by longer-term treatment strategies. Arch Gen Psychiatry. 2004;61:1097-1103.

10. Bulloch AG, Patten SB. Non-adherence with psychotropic medications in the general population. Soc Psychiatry Psychiatric Epidemiol. 2010;45:47-56.

11. Demyttenaere K. Compliance during treatment with antidepressants. J Affect Disord. 1997;43:27-39.

12. Feetam C. Medicine taking behaviour in depression - Part 2. Prog Neurol Psychiatry. 2009;13:30-33.

13. Demyttenaere K, Enzlin P, Dewé W, et al. Compliance with antidepressants in a primary care setting, 1 : beyond lack of efficacy and adverse events. J Clin Psychiatry. 2001;62:30-33.

14. Kwon A, Bungay KM, Pei Y, et al. Antidepressant use: concordance between self-report and claims records. Med Care. 2003;41:368-374.

15. Myers ED, Branthwaite A. Out-patient compliance with antidepressant medication. Br J Psychiatry. 1992;160:83-86.

16. Shi L, Liu J, Fonseca V, Walker P, Kalsekar A, Pawaskar M. Correlation between adherence rates measured by MEMS and selfreported questionnaires: a meta-analysis. Health Qual Life Outcomes. 2010;8:99.

17. Demyttenaere K, Enzlin P, Dewé W, et al. Compliance with antidepressants in a primary care setting, 2: the influence of gender and type of impairment. J Clin Psychiatry. 2001;62:34-37.
18. Demyttenaere K. Compliance and acceptance in antidepressant treatment. Int J Psychiatry Clin Pract. 2001;5:29-35.

19. Sher I, McGinn L, Sirey JA, Meyers V. Effects of caregivers' perceived stigma and causal beliefs on patients' adherence to antidepressant treatment. Psychiatr Serv. 2005;56:564-569.

20. Sirey JA, Bruce ML, Alexopoulos GS, Perlick DA, Friedman SJ, Meyers BS. Perceived stigma and patient-rated severity of illness as predictors of antidepressant drug adherence. Psychiatr Serv. 2001;52: $1615-1620$.

21. Aikens JE, Nease DE, Nau DP, Klinkman MS, Schwenk TL. Adherence to maintenance-phase antidepressant medication as a function of patient beliefs about medication. Ann Fam Med. 2005;3:23-30.

22. Akincigil A, Bowblis JR, Levin C, Walkup JT, Saira J, Crystal S. Adherence to antidepressant treatment among privately insured patients diagnosed with depression. Med Care. 2007;45:363-369.

23. Ayalon L, Areán PA, Alvidrez J. Adherence to antidepressant medications in black and Latino elderly patients. Am J Geriatr Psychiatry. 2005; 13:572-580.

24. Bull SA, Hu XH, Hunkeler EM, et al. Discontinuation of use and switching of antidepressants influence of patient-physician communication. JAMA. 2002;288:1403-1409.

25. Chen SY, Hansen RA, Gaynes BN, Farley JF, Morrissey JP, Maciejewski ML. Guideline-concordant antidepressant use among patients with major depressive disorder. Gen Hosp Psychiatry. 2010;32:360-367.

26. Crown WH, Treglia M, Meneades L, White A. Long-term costs of treatment for depression: impact of drug selection and guideline adherence. Value Health. 2001;4:295-307.

27. Donohue JM, Berndt ER, Rosenthal M, Epstein AM, Frank RG. Effects of pharmaceutical promotion on adherence to the treatment guidelines for depression. Med Care. 2004;42:1176-1185.

28. Granger AL, Fehnel SE, Hogue SL, Bennett L, Edin HM. An assessment of patient preference and adherence to treatment with Wellbutrin SR: a web-based survey. J Affect Disord. 2006;90:217-221.

29. Keeley R, Smith M, Miller J. Somatoform symptoms and treatment nonadherence in depressed family medicine outpatients. Arch Fam Med. 2000;9:46-54.

30. Keeley RD, Davidson AJ, Crane LA, Matthews B, Pace W. An association between negatively biased response to neutral stimuli and antidepressant nonadherence. J Psychosom Res. 2007;62:535-544.

31. Lin HC, Erickson SR, Balkrishnan R. Antidepressant utilization, adherence, and health care spending in the United States: the case of MDD patients 2000-2007. Health Outcomes Res Med. 2011;2:e79-e89.

32. Madsen JW, McQuaid JR, Craighead WE. Working with reactant patients: are we prescribing nonadherence? Depress Anxiety. 2009;26:129-134.

33. McLaughlin T, Hogue SL, Stang PE. Once-daily bupropion associated with improved patient adherence compared with twice-daily bupropion in treatment of depression. Am J Ther. 2007;14:221-225.

34. Merrick EL, Hodgkin D, Panas L, Soumerai SB, Ritter G. Is customization in antidepressant prescribing associated with acute-phase treatment adherence? J Pharm Health Serv Res. 2012;3:11-16.

35. Pfeiffer PN, Ganoczy D, Zivin K, Valenstein M. Benzodiazepines and adequacy of initial antidepressant treatment for depression. J Clin Psychopharmacol. 2011;31:360-364.

36. Olfson M, Marcus SC, Tedeschi M, Wan GJ. Continuity of antidepressant treatment for adults with depression in the United States. Am J Psychiatry. 2006;163:101-108.

37. Sanglier T, Saragoussi D, Milea D, Auray JP, Valuck RJ, Tournier M. Comparing antidepressant treatment patterns in older and younger adults: a claims database analysis. J Am Geriatr Soc. 2011;59: 1197-1205.

38. Stang P, Suppapanaya N, Hogue SL, Park D, Rigney U. Persistence with once-daily versus twice-daily bupropion for the treatment of depression in a large managed-care population. Am J Ther. 2007;14:241-246.

39. Voils CI, Steffens DC, Flint EP, Bosworth HB. Social support and locus of control as predictors of adherence to antidepressant medication in an elderly population. Am J Geriatr Psychiatry. 2005;13:157-165. 
40. White TJ, Vanderplas A, Ory C, Dezii CM, Chang E. Economic impact of patient adherence with antidepressant therapy within a managed care organization. Dis Manag Health Outcome. 2003;11:817-822.

41. Woolley SB, Fredman L, Goethe JW, Lincoln AK, Heeren T. Hospital patients' perceptions during treatment and early discontinuation of serotonin selective reuptake inhibitor antidepressants. J Clin Psychopharmacol. 2010;30:716-719.

42. Wu CH, Erickson SR, Piette JD, Balkrishnan R. The association of race, comorbid anxiety, and antidepressant adherence among Medicaid enrollees with major depressive disorder. Res Social Adm Pharm. 2012;8:193-205

43. Burra TA, Chen E, Mclntyre RS, Grace SL, Blackmore ER, Stewart DE. Predictors of self-reported antidepressant adherence. Behav Med. 2007;32:127-134

44. Cohen NL, Ross EC, Bagby RM, Farvolden P, Kennedy SH. The 5-factor model of personality and antidepressant medication compliance. Can J Psychiatry. 2004;49:106-113.

45. Roca M, Armengol S, Monzón S, Salva J, Gili M. Adherence to medication in depressive patients. J Clin Psychopharmacol. 2011;31: 541-543.

46. Oller-Canet S, Lacasta-Tintorer D, Castro Rodríguez JI, et al. Do depressed patients comply with treatments prescribed? A crosssectional study of adherence to the antidepressant treatment. Actas Esp Psiquiatr. 2011;39:288-293.

47. Hung CI, Wang S, Liua CY, Hsua SC, Yange CH. Comorbidities and factors related to discontinuation of pharmacotherapy among outpatients with major depressive disorder. Compr Psychiatry. 2011;52:370-377.

48. Yeh MY, Sung SC, Yorker BC, Sun CC, Kuo YL. Predictors of adherence to an antidepressant medication regimen among patients diagnosed with depression in Taiwan. Issues Ment Health Nurs. 2008;29:701-717.

49. Maidment R, Livingston G, Katona C. 'Just keep taking the tablets': adherence to antidepressant treatment in older people in primary care. Int J Geriatr Psychiatry. 2002;17:752-757.

50. Russell J, Kazantzis N. Medication beliefs and adherence to antidepressants in primary care. N Z Med J. 2008;121:14-20.
51. Zivin K, Kales HC. Adherence to depression treatment in older adults: a narrative review. Drugs Aging. 2008;25:559-571.

52. Bosworth HB, Voils CI, Potter GC, Steffens DC. The effects of antidepressant medication adherence as well as psychosocial and clinical factors on depression outcome among older adults. Int $J$ Geriatr Psychiatry. 2008;23:129-134.

53. Ibrahim S, Zhang A, Mercer M, Baughman M, Kwoh C. Inner city African-American elderly patients' perceptions and preferences for the care of chronic knee and hip pain: findings from focus groups. J Gerontol. 2004;59:1318-1322.

54. Jimenez DE, Bartels SJ, Cardenas V, Dhaliwal SS, Alegría M. Cultural beliefs and mental health treatment preferences of ethnically diverse older adult consumers in primary care. Am J Geriatr Psychiatry. 2012;20:533-542.

55. Cooper LA, Gonzales JJ, Gallo JJ, et al. The acceptability of treatment for depression among African-American, Hispanic, and white primary care patients. Med Care. 2003;41:479-489.

56. Givens JL, Houston TK, Van Voorhees BW, Ford DE, Cooper LA. Ethnicity and preferences for depression treatment. Gen Hosp Psychiatry. 2007;29:182-191.

57. Aranda JM, Vazquez R. Awareness of hypertension and diabetes in the Hispanic community. Clin Cornerstone. 2004;6:7-13.

58. Townsend MS. Patient-driven education materials: low-literate adults increase understanding of health messages and improve compliance. Nurs Clin North Am. 2011;46:367-378.

59. Demyttenaere K. Noncompliance with antidepressants: who's to blame? Int Clin Psychopharmacol. 1998;13:19-25.

60. Demyttenaere K, Adelin A, Patrick M, Walthere D, Katrien DB, Sangeleer M. Six-month compliance with antidepressant medication in the treatment of major depressive disorder. Int Clin Psychopharmacol. 2008;23:36-42.

61. Brook OH, van Hout HPJ, Stalman WAB, de Haan M. Nontricyclic antidepressants: predictors of nonadherence. J Clin Psychopharmacol. 2006;26:643-647
Patient Preference and Adherence

\section{Publish your work in this journal}

Patient Preference and Adherence is an international, peer-reviewed, open access journal focusing on the growing importance of patient preference and adherence throughout the therapeutic continuum. Patient satisfaction, acceptability, quality of life, compliance, persistence and their role in developing new therapeutic modalities and compounds to

\section{Dovepress}

optimize clinical outcomes for existing disease states are major areas of interest. This journal has been accepted for indexing on PubMed Central. The manuscript management system is completely online and includes a very quick and fair peer-review system. Visit http://www.dovepress.com/ testimonials.php to read real quotes from published authors. 